ISSN electrónico: 1885-5210

DOI: https://doi.org/10.14201/rmc2020161715

\title{
TÚ A HARVARD Y YO A PRINCENTON. DE LA DEPRESIÓN A LA ESQUIZOFRENIA
}

\section{You to Harvard and I to Princeton. From depression to schizophrenia}

\author{
Ma Adolfina RUÍZ MARTíNEZ; Sebastián PERALTA GALISTEO; Herminia CASTÁN URBANO \\ Departamento de Farmacia y Tecnología Farmacéutica. Facultad de Farmacia. Universidad de Granada (España). \\ e-mail: adolfina@ugr.es
}

Fecha de recepción: 5 de abril de 2019

Fecha de aceptación: 23 de mayo de 2019

Fecha de publicación: 15 de marzo de 2020

\section{Resumen}

Hasta hace poco tiempo las enfermedades mentales eran tratadas como un todo, sin diferenciación alguna; las investigaciones realizadas en estos últimos años han resaltado las diferencias existentes entre algunas de ellas, de tal forma que hoy en día existen tratamientos específicos para las patologías descritas. El objetivo principal de este trabajo, es el de mostrar dos enfermedades diferentes pero que en muchos casos se confunden, depresión y esquizofrenia. Patologías que pueden tener como desencadenantes, situaciones muy diversas y que requieren un tratamiento farmacológico diferente. Para ello, se realizará un análisis de dos películas, Prozac Nation (2001) de Erik Skjoldbjaerg y Una mente maravillosa (2001) de Ron Howard que reflejan las vivencias reales de dos personajes; se analizará la sociedad en la que conviven y el entorno que les rodea, pero resaltando muy especialmente el apartado de la farmacoterapia, que medicamentos se utilizan, si es correcto su uso, los efectos adversos que presentan... para de esta forma poder prever si la visión de la película tiene carácter formativo en este tipo de temática.

Palabras clave: depresión; esquizofrenia; medicamentos; Prozac ${ }^{\circledR}$; insulina; torazina.

\section{Summary}

Until recently mental diseases were treated as a whole, without any differentiation. Recent researchs have high-lighted the differences between some of them, so consequently there are specific treatments for these pathologies today. The main objective of this work is to show two different diseases which in many cases are confused: depression and schizophrenia. These pathologies can have as triggers situations very diverse and that require different treatment. For the analysis, we are going to use two films: Prozac Nation (2001) by Erik Skjoldbjaerg and A beautiful mind (2001) by Ron Howard, which reflect the real experiences of two characters; the society in which they live and the environment around them, but especially focusing in the pharmacotherapy area, medications used, its correct use, the adverse effects and so on; in order to be able to decide if the vision presented by films has a formative character in these diseases.

Key words: depression; schizophrenia; drugs; Prozac ${ }^{\circledR}$; insulin; thorazine. 


\section{Introducción}

Hoy en día los medios de comunicación poseen una gran influencia sobre la sociedad, de forma que las personas obtienen datos a través de dichos medios y luego crean una opinión o conducta en función de lo visto y escuchado. El cine es un medio de comunicación que a día de hoy tiene mucho peso en la sociedad, y puede ser utilizado como un método educativo ${ }^{1,2}$. Según Ferrándiz y col. ${ }^{3}$, esto sería así ya que el cine consigue aunar de manera impecable el arte, el entretenimiento y la capacidad para transmitir una idea, pero es que, además, se consume de forma masiva, lo que resulta fundamental. El cine mejorará su capacidad como herramienta de aprendizaje si tras la proyección de una película se organiza un proceso de estudio y análisis de su contenido, pero se sabe que es de gran importancia el hacer una selección correcta de una película, acorde con el tema a tratar ${ }^{4,5}$.

En este trabajo se analizan dos películas, [Prozac Nation (2001) de Erik Skjoldbjaerg y Una mente maravillosa / A Beautiful Mind (2001) de Ron Howard], que retratan a dos personajes reales, Elizabeth Wurtzel y John Nash, que han sufrido depresión y esquizofrenia respectivamente. Los dos filmes reflejan la vida, enfermedad y tratamiento terapéutico de los personajes centrales, así como de la influencia de su entorno. Nuestro objetivo es realizar un análisis de la temática presentada en la película, resaltando muy especialmente el apartado de tratamientos farmacológicos, ver que medicamentos se utilizan, si es correcto su uso, los efectos adversos que presentan... para de esta forma poder prever si la visión de la película tiene carácter formativo en este tipo de temática.

\section{Depresión: Prozac Nation (2001)}

\section{Ficha técnica}

Título original: Prozac Nation.

País: Estados Unidos.

Año: 2001.

Director: Erik Skjoldbjaerg.

Música: Nathan Larson.

Fotografía: Erling Thurmann-Andersen.

Montaje: James Lyons.

Guión: Larry Gross, Frank Deasy (Novela: Elizabeth Wurtzel).

Intérpretes: Christina Ricci, Anne Heche, Michelle Williams, Jason Biggs, Rob Freeman, Jesse Moss, Jonathan Rhys Meyers, Cindy Lentol, Jessica Lange, Devon Diep,...
Color: color.

Duración: 99 minutos.

Género: drama.

Idioma original: inglés.

Productora: Coproducción Estados Unidos-Alemania; Miramax / Millennium Films / Cinerenta Medienbeteiligungs KB.

Sinopsis: durante el primer curso en la prestigiosa universidad de Harward, una joven (Christina Ricci) tiene que enfrentarse a una profunda depresión.

Enlace: https://www.filmaffinity.com/es/film463835.html https://www.imdb.com/title/tt0236640

\section{Trailer}

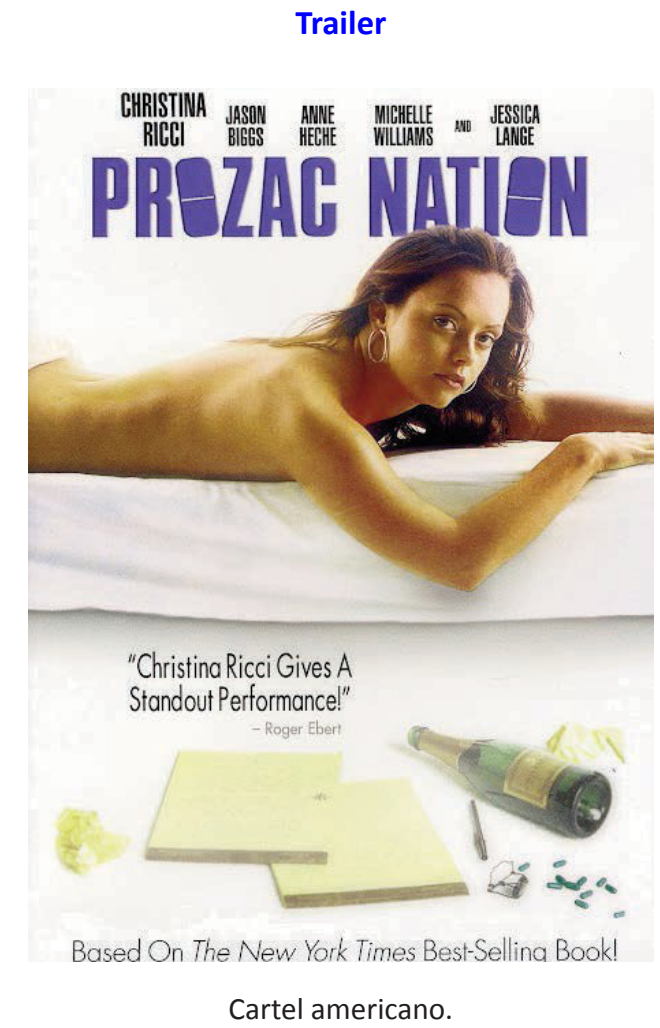

El tema de la película seleccionada Prozac Nation (2001) de Erik Skjoldbjaerg, está basada en una historia real, la vida de Elizabeth Wurtzel (Lizzie) (Christina Ricci), una joven promesa del periodismo, siendo este el motivo por el cual le conceden una beca para ir a la Universidad de Harvard. En esta película, la protagonista, debido al divorcio de sus padres padece una depresión con 
trastornos de personalidad bipolar, que la lleva a situaciones de euforia y consumo de drogas, antecedentes a tener en cuenta a la hora de diagnosticar la depresión.

En la película, se analiza muy bien el papel de la psiquiatra, la doctora Sterling (Anne Heche) que va haciendo terapia con Lizzie para hacer un buen diagnóstico de la depresión (Foto 1), es este apartado, el que se trata de forma más extensa, con visión de los efectos que se producen, la influencia en y de la sociedad, del consumo de medicamentos para esta alteración, etc.

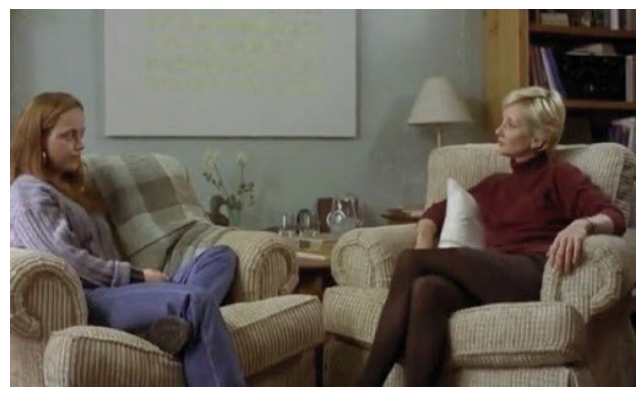

Foto 1. Terapia oral entre psiquiatra y paciente.

La protagonista ha crecido en una familia de padres divorciados desde que ella era muy pequeña, por lo que ha vivido sólo con su madre, ya que su padre se desentendió de ella. Al vivir en esta situación, la madre se convierte en una súper protectora y agobiante, planificando el futuro de la niña para paliar sus frustraciones. Siempre fue una niña un poco particular, sin muchos amigos, y desde muy pequeña su madre la apuntó a clases de teatro, la llevaba a conciertos, cine, museos... y con tan solo siete años comenzó a escribir, por lo que la madre dirige todos sus esfuerzos para encaminarla hacia el mundo del periodismo.

Su inicio en la universidad fue de maravilla. Conoció amigos y escribía sin problemas al mismo tiempo que se divertía, y por ello le conceden, en su primer año en Harvard, el "Premio de periodismo universitario»; gracias a dicho galardón, el editor de la revista Rolling Stone le propone ser la encargada de cubrir los reportajes sobre todos los grupos de música, ya que es de las pocas personas que va a los conciertos para después hacer críticas. De este modo, parece que todo va sobre ruedas hasta que su padre reaparece con otra mujer y el mundo se le cae encima de nuevo. No se inspira para escribir, por lo que se introduce en el mundo de las drogas, sexo y alcohol (Foto 2). Con todo ello pretende mantenerse despierta e intentar concentrarse en los artículos para la revista, sin éxito alguno.

Durante este periodo de tiempo, tiene una crisis de ansiedad estando con sus amigos y ellos deciden llevarla a terapia con una psiquiatra, -la doctora Sterling-; la protagonista entra en una fase de depresión cuando una mañana se levanta con miedo a vivir, dice que se ahoga en una ola negra y cuanto más angustiada está, su amiga y su novio la ignoran, es por ello que poco a poco va aceptando ir a terapia con la psiquiatra, además conoce a otro chico, con el que parece estar a gusto y contenta, pero la desconfianza y el miedo al rechazo, sobrevuelan en su cabeza, a pesar de saber que es debido a la historia del divorcio de sus padres. La psiquiatra le propone comenzar un tratamiento farmacológico, a base de un antidepresivo oral, la fluoxetina, comercializada con el nombre de $\operatorname{Prozac}^{\circledR}$, advirtiéndole que los efectos comenzaran a percibirse de 2 a 6 semanas después del inicio del tratamiento ${ }^{6}$.

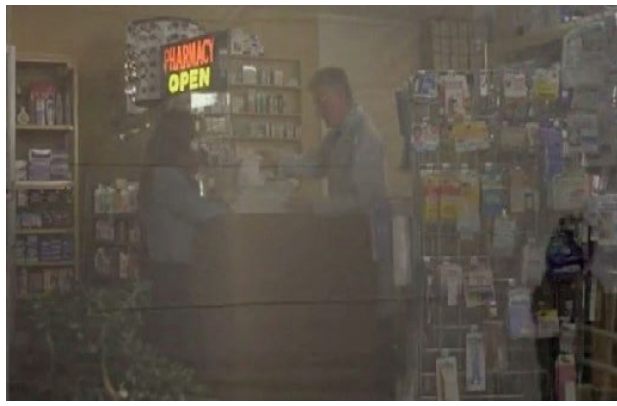

Foto 2. Adquisición de medicamentos.

Lizzie acepta el tratamiento y después de un tiempo, observa que un elevado porcentaje de la población de Estados Unidos vive inmersa en la depresión. Ella se siente bien, aunque también sabe que no es "ella», que está en tratamiento y que todo está enmascarado y vuelve a tener una crisis con ideas de suicidio en la consulta de la psiquiatra. Esta idea no llega a ningún lugar y definitivamente empieza a salir a flote cuando asimila que todo lo malo forma parte de un lento proceso de recuperación y que, aunque las pastillas no eran la salvación, le daban un respiro para poder reanudar su trabajo, el poder escribir.

Siete años más tarde (1994), Elizabeth Wurtzel publica el que llegará a ser best-seller internacional "Nation Prozac», autobiografía de la protagonista.

La película no llego en España a la gran pantalla pero si se ha editado en DVD. 


\section{Características del tratamiento}

El medicamento utilizado en esta película, como bien menciona su propio nombre, es $\operatorname{Prozac}^{\circledR}$, cuyo principio activo es fluoxetina. La protagonista toma el medicamento por vía oral en forma de capsulas gelatinosas duras. Tan solo en Estados Unidos se prescriben más de trescientos millones de prozac ${ }^{\circledast}$ y otros antidepresivos al año.

Los efectos farmacológicos que produce dicho medicamento es el aumento de los niveles de serotonina cerebrales, debido a que las personas con depresión poseen estos niveles bajos (Foto 3).

A pesar de ser un antidepresivo (ISRS) ${ }^{7}$, necesita un periodo de latencia para comenzar a apreciar los efectos del mismo, por lo que puede parecer una controversia, ya que la paciente una vez comenzado el tratamiento vuelve a sufrir una crisis depresiva con ideas de suicidio. En la película, este tema es bien considerado, puesto que la información que la psiquiatra le proporciona es adecuada.

En una primera instancia, la protagonista se encuentra en un periodo de incumplimiento terapéutico al no observar los efectos farmacológicos de inmediato.

El tratamiento no debe abandonarse a menos que lo indique un profesional a pesar de sentirse mejor, ya que si lo hace anticipadamente puede notar síntomas como: dolor de cabeza, mareos, náuseas y/o vómitos, hormigueo, pesadillas o insomnio, agitación, cansancio y ansiedad.

La psiquiatra prescribe antidepresivos, contándole a la paciente que los efectos tardan de 2 a 6 semanas en aparecer, lo que coincide con la realidad, así como las ideas de suicidio que presenta la protagonista debido al periodo tan corto de tiempo transcurrido desde el inicio del tratamiento. Al igual que en los apartados anteriores, la película refleja con veracidad estos hechos, asi como los efectos que aparecen tras la medicación.

Podemos señalar que bajo nuestro punto de vista, la película trata de forma muy acertada el tema de la depresión como enfermedad, con descripción de efectos que produce y la implicación en la sociedad. El tema de la administración de medicamentos se analiza de forma breve, pero nítida, ya que queda perfectamente claro como el incumplimiento terapéutico agrava la enfermedad, y como es necesario no dejar de tomar los medicamentos el tiempo necesario (periodo de latencia) para poder apreciar sus efectos, al igual que se demuestra que es necesario un tratamiento para aminorar o paliar la depresión, enfermedad que cada día está mas presente en nuestro entorno, pero que llega a ser una "gran desconocida» para muchos. Por el contrario, en la película no podemos apreciar la posología del fármaco administrado, tema importantísimo a tener en cuenta para cada paciente y sus circunstancias.

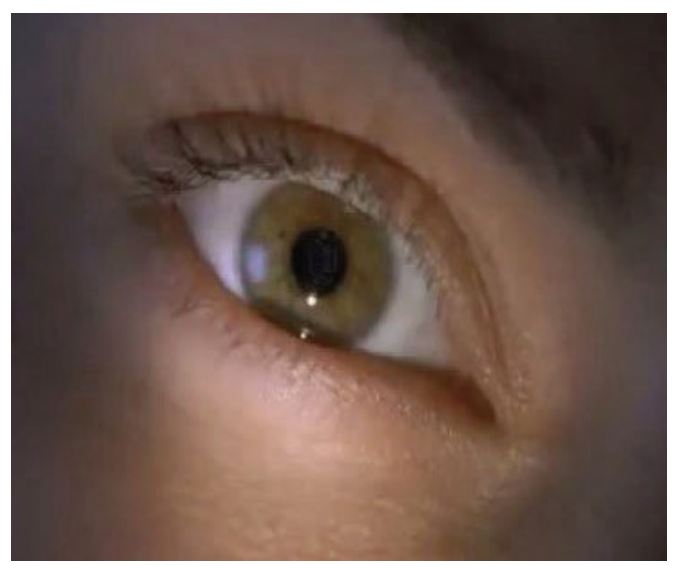

Foto 3. Detección de algunos efectos adversos de los medicamentos.

Tras el análisis de todo ello, podemos destacar el importante papel que desempeñan todos los especialistas de la salud a la hora del diagnóstico, y de la adherencia a los tratamientos farmacológicos, por lo que estos deben saber transmitir y hablar con el paciente o familiares, sobre la enfermedad así como inculcarles la importancia de tomar su medicación.

Esquizofrenia: Una mente maravillosa (2001)

\section{Ficha técnica}

Título original: A Beautiful Mind.

País: Estados Unidos.

Año: 2001.

Dirección: Ron Howard. Música: James Horner. Fotografía: Roger Deakins.

Montaje: Daniel P. Hanley, Mike Hill.

Guión: Akiva Goldsman (Libro: Sylvia Nasar). Intérpretes: Russell Crowe, Jennifer Connelly, Ed Harris, Paul Bettany, Adam Goldberg, Judd Hirsch, Christopher Plummer, Josh Lucas, Anthony Rapp, Austin Pendleton, Jason Gray Stanford, Vivien Cardone, Ron Howard.

Color: color.

Duración: 130 minutos.

Género: drama.

Idioma original: inglés. 
Productora: DreamWorks SKG / Universal Pictures / Imagine Entertainment.

Sinopsis: obsesionado con la búsqueda de una idea matemática original, el brillante estudiante John Forbes Nash (Russell Crowe) llega a Princeton en 1947 para realizar sus estudios de postgrado. Es un muchacho extraño y solitario, al que sólo comprende su compañero de cuarto (Paul Bettany). Por fin, Nash esboza una revolucionaria teoría y consigue una plaza de profesor en el MIT. Alicia Lardé (Jennifer Connelly), una de sus alumnas, lo deja fascinado al mostrarle que las leyes del amor están por encima de las de las matemáticas. Gracias a su prodigiosa habilidad para descifrar códigos es reclutado por Parcher William (Ed Harris), del departamento de Defensa, para ayudar a los Estados Unidos en la Guerra Fría contra la Unión Soviética.

Enlace: https://www.filmaffinity.com/es/film326587.html https://www.imdb.com/title/tt0268978

\section{Trailer}

Se analiza en este caso una película que trata sobre la esquizofrenia, Una mente maravillosa (2001) de Ron Howard, identificando sus fortalezas y debilidades, como herramienta pedagógica.

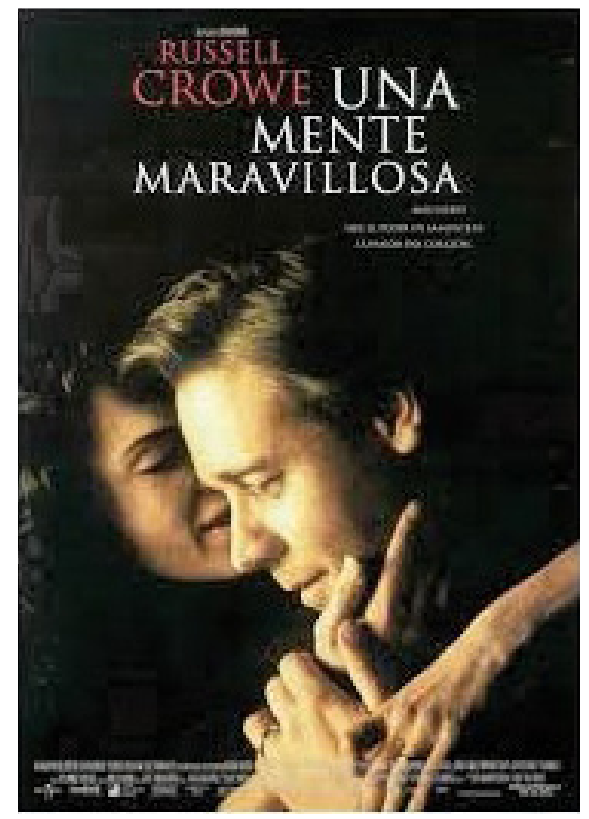

Cartel español.
Esta película, cuyo director es Ron Howard, es un drama biográfico estadounidense rodado en el año 2001, basada en el libro del mismo nombre de la escritora Sylvia Nasar, el cual relata la historia de John Forbes Nash, ganador del Premio Nobel de Economía en 1994. También es importante destacar, que la película fue ganadora del óscar a la mejor película en el 2001.

En el film, se relata la vida de John Nash y su lucha contra la esquizofrenia. Al inicio de la película se nos muestra a un joven John Nash (Russell Crowe), el cual comienza la carrera de matemáticas en la prestigiosa Universidad de Princeton. Desde el primer instante nos damos cuenta que carece de capacidades para sociabilizarse (Foto 4), y como ya en esta etapa comienza a tener alucinaciones, puesto que su compañero de habitación Charles Herman (Paul Bettany), no es real, sino un producto de su imaginación.

A pesar de todo, John es un matemático excelente, llevando a cabo una Teoría de Juegos no Cooperativos, que le daría su puesto en el Massachusetts Institute of Technology (MIT) y que además, encontraría aplicaciones en el campo de la economía, política e incluso en el campo de la biología evolutiva. Trabajando en el MIT, es llamado al pentágono, donde se le pide descifrar un código para el ejército, a partir de este momento, las alucinaciones de John irán en aumento, apareciendo una nueva, William Parcher (Ed Harris), quien supuestamente le dará una misión realmente importante a John, que consistirá en descifrar códigos escondidos en periódicos, revistas, etc. (Foto 5). Entre tanto, John conocerá a una alumna, que posteriormente se convertirá en su esposa, Alicia Larde (Jennifer Connelly), ella tendrá un papel fundamental, en los años más duros de la enfermedad.

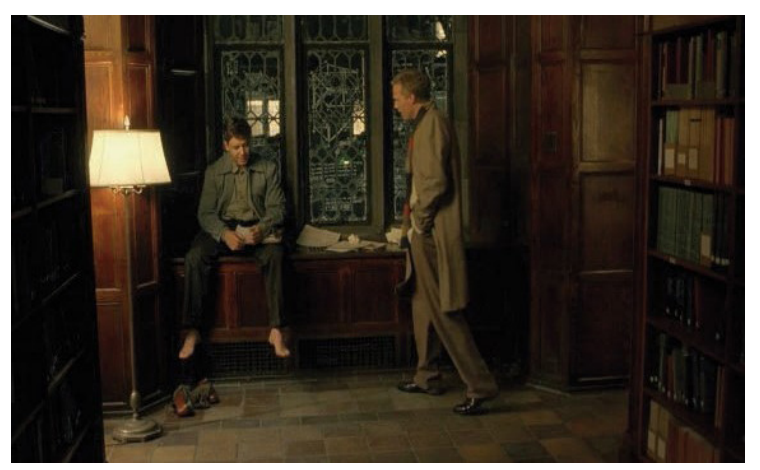

Foto 4. Aislamiento del protagonista. 


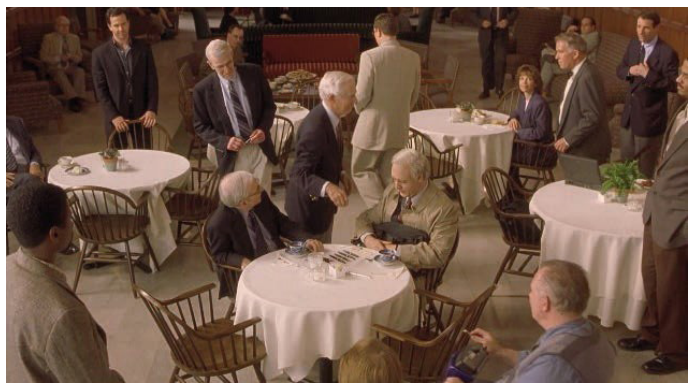

Foto 5. Alucinaciones de John.

En los años posteriores, John padece paranoias persecutorias, lo que hace que su esposa Alicia, empiece a tener sospechas sobre su estabilidad mental y decide acudir a un especialista, el Doctor Rose (Christopher Plummer). Mientras tanto, John muestra por primera vez un síntoma positivo y observable de la esquizofrenia, y es que durante una conferencia donde él participaba, habla de forma ininteligible y sin coherencia, y es en dicha conferencia, donde John será abordado por el Doctor Rose, del cual, intenta huir sin éxito.

Una vez en el psiquiátrico, John es diagnosticado de esquizofrenia paranoide, y será tratado mediante shocks de insulina durante diez semanas (https://www.youtube. com/watch?v=JevaHljSK_g). Una vez terminado el tratamiento en el psiquiátrico, regresa a casa donde seguirá con un tratamiento oral, que abandonará a causa de los efectos secundarios y del estigma social que implica el seguir este tratamiento. A causa del abandono, vuelve a tener una recaída, donde pondrá en riesgo la vida de su hijo, lo que provoca que su mujer vuelva a acudir al Doctor Rose, y se planteen el volver a internar a John, aunque finalmente, después de hablar con su marido, Alicia decide darle una oportunidad a John y probar un tratamiento diferente al farmacológico.

En las siguientes escenas, vemos a un John bastante deteriorado por la enfermedad, que camina de forma extraña y desconfiada, pero que intenta reintegrarse en la sociedad y para ello, le pide a su antiguo compañero y amigo, Martin Hansen (Josh Lucas), el cual es el jefe de departamento de matemáticas de Princeton, que lo vuelva a admitir en la Universidad, como oyente y para continuar con su trabajo, usando como despacho la biblioteca. A partir de este punto, veremos como John, vuelve a sufrir recaídas y lucha por integrarse, a pesar de las burlas de los alumnos y las miradas discriminatorias. Un día, un alumno muestra interés por el extraño hombre que pinta en las ventanas de la biblioteca y lo reconoce como John Nash, comienzan a hablar y a la charla se unirán más alumnos, esto despierta en John la posibilidad de empezar su profesión como enseñante en la universidad (Foto 6).

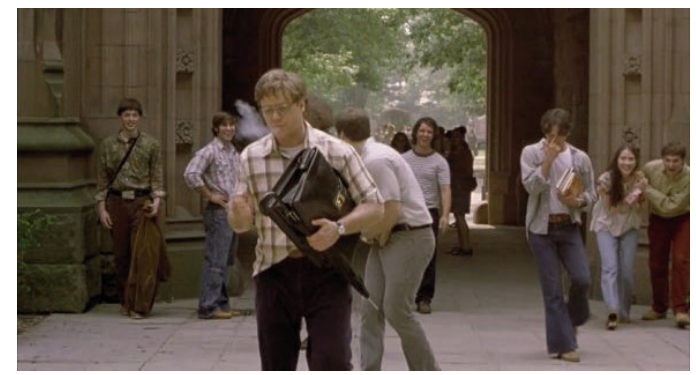

Foto 6. Vuelta a la Universidad de John.

Se produce un salto temporal, donde vemos a John Nash como profesor, el cual sigue con alucinaciones, pero ha logrado aprender a distinguir lo real de lo imaginario. Durante una de sus clases, John recibe la visita de un hombre que le dice, que ha sido seleccionado como ganador del Premio Nobel de Economía, pero que deben confirmar si todo va bien como para que pueda recibirlo, a lo que nuestro protagonista le contesta, que está con tratamiento farmacológico y que ha aprendido a ignorar las alucinaciones. En 1994, vemos a John dando su discurso como ganador del Nobel, y dando las gracias a su esposa Alicia, por estar a su lado durante la dura batalla contra su enfermedad mental.

Podemos señalar que el tema de la enfermedad, en general, está bastante bien tratado en esta película, ya que desde el inicio del film podemos observar características propias de un esquizofrénico en John Nash, como por ejemplo la falta de empatía, la dificultad para establecer relaciones sociales, así como las alucinaciones. Más adelante, veremos que en el personaje surgen otros síntomas indicativos de la enfermedad como es el habla ininteligible e incoherente (conferencia en la que participa John Nash), el comportamiento paranoico (cree que está siendo perseguido, etc.), entre otros. En cuanto a las alucinaciones, debemos observar que en ocasiones se vuelven exageradas, por ejemplo, cuando conoce a William Parcher, este lo lleva a su cuartel secreto, donde hay una tecnología espectacular y una organización enorme del gobierno.

En cuanto al tratamiento farmacológico, la película se aleja un poco de la realidad, ya que da a entender 
que John Nash es capaz de discernir entre lo real y lo imaginario, gracias a su fuerza de voluntad y a su mente. Debemos ser críticos en cuanto a este punto, ya que una de las principales causas de recaídas de esta enfermedad se debe precisamente al abandono del tratamiento farmacológico. Es cierto que en la película se muestra como John recae al dejar el tratamiento, pero justo después nos dan a entender que el busca una forma de curarse distinta al tratamiento farmacológico, y hoy en día, podemos casi afirmar que es imposible o realmente complicado. De hecho, el propio John Nash, ha matizado que esta parte de la película no es realmente correcta, y que por esas fechas estaba siendo tratado con antipsicóticos atípicos ${ }^{7}$.

Otro aspecto que viene tratado en la película y que se hace de forma acertada y correcta, es la estigmatización que sienten los enfermos de esquizofrenia, y como son marginados o cuestionados por la sociedad, lo que provoca en ellos vergüenza, generando un motivo para querer abandonar el tratamiento. Además, en el film se muestra la importancia de la reintegración social del enfermo para su recuperación y también la importancia que tiene desde el punto de vista de los familiares y cuidadores, los cuales se sienten aliviados al ver que el enfermo puede llevar una vida relativamente normal.

A medida que la película avanza, vemos que el comportamiento de John va evolucionando, de forma que se va alejando cada vez más de la realidad y desarrolla la idea delirante de que está siendo perseguido por espías rusos, es decir, en general vemos que su comportamiento pasa de ser algo peculiar a ser "extraño». Cuando llegamos a este punto de la película, se nos descubre que John padece esquizofrenia, y nosotros los espectadores achacamos ese comportamiento extravagante que veíamos anteriormente a la enfermedad. No obstante, ¿realmente es este un comportamiento habitual en pacientes diagnosticados con esquizofrenia? Para nosotros la respuesta en general es sí, ya que la esquizofrenia se caracteriza por presentar síntomas positivos en los que incluimos las ideas delirantes, las alucinaciones y el pensamiento desorganizado; ; pero también se caracteriza por presentar una serie de síntomas negativos ${ }^{9}$, entre los que cabe destacar el aplanamiento afectivo (la falta de empatía, la inexpresividad en la cara, etc.), la alogia (el paciente carece de espontaneidad y presenta una disminución del flujo de la conversación), el aislamiento, etc. Una vez que somos conocedores de estos datos, podemos dar una explicación al comportamiento peculiar que presenta John desde el inicio de la película, típico de un enfermo esquizofrénico.

Rev. Med. Cine. 2020; 16(1), 7-15
En base a lo expuesto, todos estos síntomas que acabamos de mencionar son vistos en John Nash durante la película. Como las alucinaciones e ideas delirantes son fáciles de observar por el espectador no especializado, no pondremos ningún ejemplo sobre ello, pero sí que sería satisfactorio poner un ejemplo de pensamiento desorganizado; hay una escena donde John se encuentra dando una conferencia, la cual se ve interrumpida por la entrada del Doctor Rose, y en dicha escena podemos observar como John salta de un tema a otro sin seguir un hilo coherente del discurso.

De forma general podemos señalar que la película se ajusta a la realidad, ya que el mismísimo John Forbes Nash, participó en su rodaje, haciendo puntualizaciones y dando consejos tanto al director Ron Howard como al actor que haría de él, Russell Crowe. Pero no todo se ajusta cien por cien a la realidad, sino que muchas situaciones son dulcificadas, como por ejemplo, en la película se muestran los intentos de John por reintegrarse en el mundo de la universidad, vemos como sufre crisis y como se burlan de él los alumnos, pero la realidad es que durante los años setenta y ochenta, Nash se convierte en un marginado social, que deambula por la universidad con delirios, mendigando, etc. Con el paso del tiempo es cierto que consigue controlar la enfermedad, lo que le permitirá recuperar en parte su vida cotidiana.

Hay otro aspecto de la película que no se ajusta a los hechos reales, y es el tema de la hospitalización, en la película vemos como John es hospitalizado una única vez y que, a pesar de tener una nueva crisis tras abandonar la medicación, su mujer decide confiar en él y no volver a internarlo. Esto no fue así, ya que John fue hospitalizado en diversas ocasiones, siendo algunas hospitalizaciones en contra de su voluntad y autorizadas por Alicia Larde, su esposa.

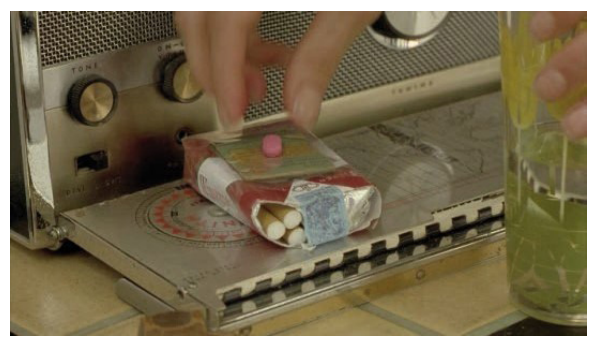

Foto 7. Medicamento utilizado por el protagonista.

Asimismo, en la película la recuperación de John Nash se lleva a cabo gracias a su «fuerza de voluntad», 
aspecto totalmente alejado de la realidad y es que el propio John Nash hizo una declaración sobre este tema, aclarando que, por aquella época, él siempre estuvo en tratamiento con antipsicóticos atípicos (Foto 7). Es cierto, que John Nash, no supo manejar en ocasiones la fama, e hizo ciertas declaraciones contradictorias con respecto a este tema, pero debemos saber, que una enfermedad como la esquizofrenia debe ser tratada correctamente y es el abandono del tratamiento lo que en la mayoría de ocasiones provoca la recaída de los pacientes ${ }^{10}$.

\section{Características sobre los medicamentos que se utilizan}

En una de las escenas del film, vemos como John huye del Doctor Rose, tras una breve persecución consiguen atrapar a nuestro asustado protagonista y lo sedan utilizando Torazina (Clorpromazina) en solución inyectable. Algunos autores ${ }^{11}$ han indicado que la clorpromazina a finales de la década de los cuarenta era utilizada como sedante, en casos de estados de agitación psicomotriz. Ahora en la actualidad, la clorpromazina todavía encuentra uso como sedante en algunos casos de psicosis maniaco - depresiva y de ansiedad grave ${ }^{12}$.

En la película, cuando John ya se encuentra hospitalizado en el psiquiátrico, vemos una escena donde se le administra una elevada dosis de insulina, lo que le provoca un estado convulsivo (Foto 8). A continuación, en la siguiente escena, se nos muestra al Doctor Rose hablando con Alicia sobre el tratamiento, diciéndole que éste deberá ser llevado a cabo cinco veces a la semana durante diez semanas. En la actualidad esta terapia esta totalmente en desuso, a pesar de que en la película se emplea.

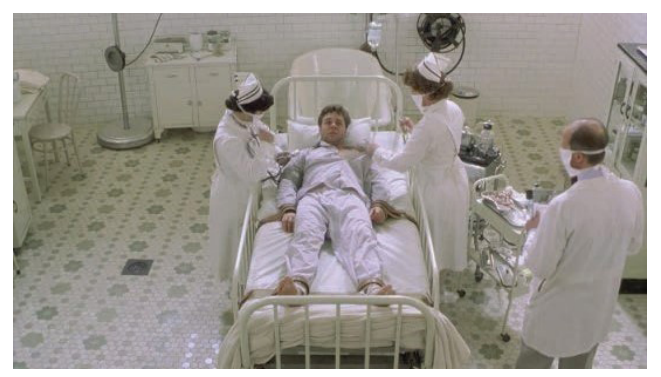

Foto 8. Tratamiento hospitalario con insulina.

Sabemos que actualmente la insulina se utiliza en el tratamiento de la diabetes, pero no siempre fue así, ya que a principios del siglo XX con los primeros experimentos de Sakel, esta hormona fue empleada en el campo de la psiquiatría ${ }^{13,14}$. Algunos estudios realizados, refieren como la administración de grandes dosis de insulina, produce en los pacientes esquizofrénicos un cambio en su carácter, de forma que se elaboró una técnica que se realizaría en cuatro fases, con el fin de curar dicho trastorno ${ }^{15}$.

Detallando las fases anteriormente mencionadas, la primera fase consiste en ir administrando dosis crecientes de insulina hasta conseguir el primer shock hipoglucémico, en la segunda fase el objetivo era lograr fuertes shocks hipoglucémicos, la tercera fase consistía en ir alternando días de choque con días de descanso, y en la cuarta y última fase, lo que se hacía era ir disminuyendo paulatinamente las dosis de insulina. La duración del tratamiento oscilaba entre los 3 y 6 meses, y se consideraba que la terapia había sido exitosa si se observaban cambios conductuales en el paciente.

El tratamiento en sí, consistía en administrar dosis que oscilaban entre 24 y $300 \mathrm{U}$ de insulina por vía intravenosa o por vía subcutánea, induciendo en el paciente un coma hipoglucémico que debía aliviar los síntomas de la esquizofrenia. El paciente permanecía durante media hora o una hora en coma, hasta que se interrumpía dicho estado, mediante la administración de té o leche azucarados a través de una sonda nasofaríngea. En ocasiones era necesario administrar por vía intravenosa un suero glucosado para conseguir interrumpir el estado comatoso. Para finalizar, decir, que la terapia con insulina presentaba una serie de complicaciones, entre las que cabe destacar los problemas cardiovasculares, las encefalopatías y el daño cerebral permanente, lo que ha hecho que hoy en dia no se utilice.

Tras la hospitalización, John Nash regresa a casa donde vemos que sigue en tratamiento con unos comprimidos de color rosa. En la película no se especifica ni el principio activo, ni la posología seguida por el protagonista, pero en cuanto a ésta última podemos ver que John ha de tomar un comprimido al medio día y otros dos por la tarde noche. Además, tras una búsqueda un poco más exhausta podemos suponer, que el principio activo que se usa es la clorpromazina ${ }^{16}$, pero esta vez en una forma farmacéutica de administración oral.

\section{Conclusiones}

Consideramos al cine, como una buena herramienta de aprendizaje, no solo para el mundo de la docencia, sino también para la sociedad como medio de salud, ya que contribuye a la percepción del mundo en el que vivimos desde otra perspectiva. 


\author{
TÚ A HARVARD Y YO A PRINCENTON. DE LA DEPRESIÓN A LA ESQUIZOFRENIA \\ M A ADOLFINA RUÍZ MARTÍNEZ; SEBASTIÁN PERALTA GALISTEO; HERMINIA CASTÁN URBANO
}

Las dos películas analizadas contribuyen de forma positiva en la descripción de las dos patologías descritas, así como en los tratamientos farmacológicos empleados.

\section{Agradecimientos}

Nuestro agradecimiento a Ana Vico y Montaña Rodilla por su colaboración.

\section{Referencias}

1. Bonilla J. El cine y los valores educativos. A la búsqueda de una herramienta eficaz de formación. Pixel - Bit. Revista de Medios y Educación 2005;(26): 39-54.

2. García Sánchez JE, García Sánchez E. El cine y la literatura en la enseñanza de la medicina. Rev Med Cine 2012;8(4): 131-32.

3. Ferrándiz ML, Noguera MA, Recio MC, Terencio MC, Montesinos MC, García Arnandis I et al. Farmacinema: Recurso docente en el aprendizaje de Farmacología y Farmacia Clínica Ars Pharm 2010;(51): 69-76.

4. García Sánchez JE, García Sánchez E. Medicina, cine y educación. Rev Med Cine 2008;4(2): 39-40.

5. Segura J, Rolo I, Parrado O. Shutter Island (2010), el trauma como desencadenante de la esquizofrenia. Rev Med Cine 2019;15(1): 37-44.

6. Botto A, Acuña J, Jiménez JP. La depresión como un diagnóstico complejo. Implicancias para el desarrollo de recomendaciones clínicas. Rev Med Chile 2014;142: 1297-305.

7. Micheli L, Ceccarelli M, D’Andrea G, Tirone F. Depression and adult neurogenesis: Positive effects of the antidepres- sant fluoxetine and of physical exercise. Brain Res Bull 2018; 143(10): 181-93.

8. Tajima K, Fernández H, López-Ibor J J, Carrasco JL, DíazMarsá M. Tratamientos para la esquizofrenia. Revisión crítica sobre la farmacología y mecanismos de acción de los antipsicóticos. Actas Esp Psiquiatr 2009;37(6): 330-42.

9. Stahl S.M. Psicofarmacología esencial: Bases neurocientíficas y aplicaciones clínicas. Ariel Neurociencia. 2002. Barcelona. 10. Gutiérrez F. Tratamiento actual con antipsicóticos de la esquizofrenia. Farm Hosp 1998;22(4): 207-12.

11. Cheng $M$, Gu H, Zheng L, Wang H, Zhong Z, Wen S. Neuroleptic malignant síndrome and subsequent clozapine-withdrawal effects in a patient with refractory schizophrenia. Neuropsychiatr Dis Treat 2016;12: 695-97.

12. Cabrera R, Manzana JS, Pereira J. Cincuenta años de clorpromazina. Revista Española de Sanidad Penitenciaria. 2004;4(3): 101-13.

13. Noyce P. How chlorpromazine improved the treatment of schizophrenic patients. New Community 2017;13: 43-6.

14. Insabato L. La terapéutica por los shocks: insulina, cardiazol, electroshock. Tratamiento diacefalorraquideo. Norte de Salud Mental 2004;5(20): 74-84.

15. Benito R, Celorrio GA, Duro A, Núñez JM, y Sánchez L. Tratamientos en la psiquiatría del siglo XX. Ponencia. XXXI Congreso Nacional de Enfermería de Salud Mental, (Toledo, España) 2014.Ilustre Colegio de Enfermería de Madrid.

16. Spagna A, He G, Jin S, Gao L, Mackie MA, Tian Y, et al. Clorpromacian: Deficit of supramodal executive control of attention in schizophrenia. J Psychiatr Res 2018;97(2): 22-9.

\begin{tabular}{|l|l|}
\hline & $\begin{array}{l}\text { Ma Adolfina Ruiz Martínez. Profesora de Farmacia y Tecnología Farmacéutica. Facultad de } \\
\text { Farmacia de Granada. }\end{array}$ \\
\hline
\end{tabular}

